\title{
Relative Performance Evaluation-A Review of Recent Literature at Home and Abroad
}

\author{
Huihuang Li \\ Jinan University, Guangzhou, China \\ Email:1hhmicjnu@163.com
}

How to cite this paper: Li, H.H. (2019) Relative Performance Evaluation-A Review of Recent Literature at Home and Abroad. Modern Economy, 10, 1229-1238. https://doi.org/10.4236/me.2019.104084

Received: March 20, 2019

Accepted: April 16, 2019

Published: April 19, 2019

Copyright (C 2019 by author(s) and Scientific Research Publishing Inc. This work is licensed under the Creative Commons Attribution International License (CC BY 4.0).

http://creativecommons.org/licenses/by/4.0/

\begin{abstract}
Relative performance evaluation is an important part of performance evaluation and compensation incentive research. Following the logical relationship among the existence, influence factors and economic consequences of relative performance evaluation, this paper sorts out the frontier research at home and abroad in recent years, focusing on the analysis and summary of the selection criteria of the peer enterprises and economic consequences of relative performance evaluation. On this basis, this paper looks forward to the future research direction. This paper has certain enlightenment significance for understanding and grasping the research dynamics, focus and future research directions of relative performance evaluation.
\end{abstract}

\section{Keywords}

Relative Performance Evaluation, Influencing Factors, Economic Consequence

\section{Introduction}

The relative performance evaluation aims to eliminate the systematic part of the company's performance by determining the peer groups' performance so that the non-systematic part of the company's performance can fully reflect the manager's efforts without interference from external common shocks, thus optimizing performance evaluation and compensation incentives. This paper reviews the research on relative performance evaluation at home and abroad in recent years, tries to summarize the focus and inadequacies of cutting-edge research at home and abroad, and looks forward to future research.

In the part of the existence and influencing factors, foreign studies question the traditional industry classification standards, and propose classification criteria based on text analysis or enterprise life cycle. At the same time, scholars also examine whether management power will affect the relative performance evalu- 
ation. However, domestic research focuses on the investigation of the existence and influencing factors of relative performance evaluation based on traditional industry classification standards, but the investigation of family enterprises is different from the research based on independent enterprises abroad. In the part of economic consequences, foreign research studies are carried out in terms of organizational identity, risk decision-making, promotion incentives, and human resource conservation. Domestic research has only two studies on the impact of salary growth and salary ratcheting effect, which seems to be fewer compared with foreign countries. In the review and prospects of the research, this paper summarizes the focus and inadequacies of research at home and abroad in recent years.

Based on the deficiencies of existing research, this paper proposes four rearch directions, which are conducting the existence test from the type of sub-enterprise, further studying the reasonable selection criteria of reference enterprises, and analyzing the research framework influencing factors and further studying of the economic consequences of relative performance evaluation. This paper has certain enlightenment significance for understanding and grasping the research dynamics, focus and future research directions of relative performance evaluation.

The article is organized as follows. First, I will introduce the research on the existence and influencing factors of relative performance evaluation. Then, I will review the literature concerning economic consequences of relative performance evaluation. Finally, I will summarize the shortcomings of the existing literature and propose four research directions.

\section{The Existence and Influencing Factors of Relative Performance Evaluation}

\subsection{Foreign Literature Review}

How to set reasonable selection criteria so that the reference enterprise performance can reflect the impact of external common shocks on corporate performance is not only the focus in performance evaluation practice, but also the difficulty of empirical verification of relative performance evaluation. The existing literature is mainly based on industry classification, and further considers the annual, scale, market and other subdivided standards, using the same industry the same scale, the same industry - the same year - the same scale, the same industry - the same scale - the same market and other standards to determine the reference to corporate performance and test the existence of relative performance evaluation. However, in recent years, two representative studies related to the selection criteria of reference companies, Jayaraman et al. [1] and Drake and Martin [2], have questioned the classification criteria based on static industries. The former proposed text analysis technology to identify the reference enterprise from the perspective of the supply chain, while the latter proposes a method to identify the reference enterprise from the perspective of the life cycle.

Jayaraman et al. [1] believe that the industry classification of SIC and GICS 
may not reflect the heterogeneity of profitability and growth in the industry, and the industry classification often cannot be adjusted in time as the product market strategy changes. Therefore, the use of SIC and GICS to determine the common external impact of a company's industry may have measurement errors, and thus cannot provide evidence of the existence of RPE. If a company has similar suppliers, product markets, and customers, then the supply and demand risks it faces are similar. By defining a reference enterprise using textual analysis technology, the research finds that relative performance evaluation can eliminate the impact of common impact on company performance, and this elimination effect is enhanced with the increase of reference enterprises. It can be completely eliminated when the number of the peer firm reaches at a certain amount. At the same time, they pointed out that the key to testing the relative performance theory is to correctly define the reference enterprise.

Drake and Martin [2] extended the theory of relative performance evaluation and provided evidence that the compensation committee may select companies outside the industry based on whether they are in the same life cycle stage and exclude companies within the industry as reference companies to eliminate the systematic part of the company performance. Their research found that static industry classifications do not reflect the fact that the company is developing at a high speed. Therefore, it is difficult to determine the impact of common shocks by referring to enterprises in the traditional industry classification. The external common shocks faced by enterprises in the same life cycle are similar to each other. Therefore, incorporating companies with the same life cycle within and outside the industry as the reference group can fully eliminate the impact of common shocks.

In addition to corporate selection criteria, corporate governance characteristics are also important factors influencing relative performance evaluation. Managerial power theory argues that because executives have a lot of power, their power will also affect the design of executive compensation contracts. Dikolli [3] studied the impact of CEO power on relative performance evaluation. On the one hand, when the CEO has great power, whether or not the CEO will interfere with the choice of the reference company. On the other hand, when the CEO has greater power, whether or not the board will adopt relative performance evaluation. The study found that a more powerful CEO would eliminate some of the systemic risks by influencing relative performance evaluation, and the board may not adopt a relative performance evaluation when the performance of the same industry is expected to be high.

\subsection{Domestic Literature Review}

Considering China's special institutional background, Chinese scholars also try to use a variety of performance evaluation indicators and reference enterprise selection criteria to investigate the application of relative performance evaluation in China's listed companies under the influence of internal and external factors. $\mathrm{Hu}$ and Zhou [4] used stock return and return on assets to measure the compa- 
ny's performance, and adopted the criterion of the same industry, the same market, and the same scale to select the reference enterprise to test the existence of relative performance evaluation and the impact of the company's growth on performance evaluation. The study found that when the reference group is defined based on the same industry company and the company's performance is measured by the return on assets, there shows supporting evidence of the relative performance evaluation hypothesis. The growth of the company is negatively correlated with the use of relative performance evaluation. Chen et al. [5] used ROE and RET as performance evaluation indicators, and used the criterion of the same year, the same industry, the same property rights and the criterion of the same year, the same industry, the same property rights and the same scale to choose the reference enterprise. The study found that relative performance evaluation has not been widely used in listed companies in China. Non-state-owned enterprises are more likely to use relative performance evaluation than state-owned enterprises. This may be due to the fact that state-owned enterprise suffer from pay controls, multiple forms of incentives, and multiple tasks.

Different from the previous literature using financial performance or capital market indicators to measure corporate performance, Zhou et al. [6] used enterprise technical efficiency (measured by operating profit margin, total net profit margin and net asset return) to quantify corporate performance and adoped the criterion of the same year, the same industry and the criterion of the same year, the same industry, the same scale to select the reference enterprise, test the use of relative performance evaluation and the impact of market competition, property rights and technical efficiency on the relative performance evaluation. The study found that relative performance evaluation has not been widely used in listed companies. In addition, companies with high technical efficiency, companies within the industry which has higher level of competition, and private companies facing low market concentration are more likely to adopt relative performance evaluations. Zhang and Wang [7] examined the existence of relative performance evaluation and the impact of marketization process and the nature of corporate ownership. The research results show that the relative performance evaluation is widely used in the executive compensation contracts of listed companies in China. Compared with the less market-oriented regions, enterprises in regions with higher degree of marketization tend to use relative performance evaluation. Non-state-owned enterprises are more likely to implement relative performance evaluations in areas with higher levels of marketization than the state-owned enterprises.

All of the above studies considered the study subjects as independent companies without considering the impact of other subsidiaries on relative performance evaluation when the company was placed in a corporate group. In reality, there are a large number of corporate groups in Asian countries, and studies have not considered the impact of subsidiaries within the group. The reason for considering other subsidiaries within the group when studying the relative per- 
formance evaluation is that the subsidiaries in the group may be affected by external common shocks due to the same group, and the executives of each subsidiary may conduct a horizontal comparison of the compensation of the management within the group to assess whether the compensation of their own are fair. For the above reasons, Cai and Zheng [8] used the 2003-2011 Chinese Family Group data and found that the relative performance evaluation was widely used in the formulation of executive compensation for family subsidiaries and when the family group was non-state-owned or the marketization degree is high, the compensation of the executives of the subsidiary companies is more susceptible to the influence of other subsidiaries.

\section{Economic Consequences of Relative Performance Evaluation}

\subsection{Foreign Literature Review}

Since foreign research has widely confirmed the existence of relative performance evaluation, the focus of research has gradually turned to the economic consequences of relative performance evaluation. Foreign studies have studied the economic consequences of adopting relative performance evaluation from the aspects of organizational identity, risk selection, promotion incentives, and human resource conservation, which are more abundant than domestic ones.

Previous research focused on the impact of relative performance evaluation on executive performance, while ignoring whether relative performance evaluation would affect executives' attitudes toward the organization. Mahlendorf et al. [9] used experimental methods to study under what conditions providing relative performance information could enhance organizational support (POS). Studies have shown that when relative performance information is useful and information recipients have high levels of performance and their professional identity is strong, providing relative performance information can enhance organizational support (organizational identity, job engagement, and non-job performance).

Relative performance evaluation aims to eliminate the impact of the systemic part of the company's performance, which may have an impact on executive risk selection decisions. Park and Vrettos [10] studied two questions: First, whether the application of relative performance evaluation in the compensation contract enhances the impact of performance mix on overall risk. Second, whether relative performance evaluation will affect the relationship between performance portfolio and systemic risk and individual risk, that is, whether relative performance evaluation has a regulatory effect on enterprise risk composition. Studies have shown that when there is a lack of relative performance evaluation in the incentive design, the sensitivity of the CEO performance portfolio value to stock return volatility (external shock) will encourage the CEO to improve the overall risk through a systematic part, because this risk can be hedged by index trading. Conversely, when using relative performance evaluation in incentive design, CEOs tend to have individual risks (non-systematic risks) because relative per- 
formance assessments filter out the systematic part of company performance.

Standard agency theory and tournament theory provide a theoretical explanation for relative performance evaluation. However, equity incentives will cause managers to face stock price fluctuations and suffer from the company's systemic risk, which is inconsistent with the interpretation of standard agency theory. The premise of tournament theory is that if a company compares with another company, another company must also compare with the company. But this is not the case in the real world. Based on this, Angelis and Grinstein [11] gave an explanation of non-agent theory that if management talent is easy to transfer, companies are more inclined to use relative performance evaluation to reward managers for their talents beyond their peers (pay for talent) so that the company's human capital can be maintained.

Demeré et al. [12] argue that relative performance evaluation can provide useful information for promotion decisions, enabling the most hard-working or capable people to win promotion incentives. Relative performance evaluation strengthens the correlation between managerial efforts and their performance by reducing external factors in performance evaluation. As the results of the relative performance evaluation providing useful information for promotion, managers will strive to increase their performance to win promotion incentives. However, research also shows that the incentive effect of relative performance evaluation is positively correlated with the probability of advanced promotion. If managers believe that the chances of promotion are small, the incentive effect of relative performance evaluation will be greatly limited.

\subsection{Domestic Literature Review}

Because there are still conflicting evidences about the existence of relative performance evaluation, domestic research on the economic consequences of research relative performance evaluation is still limited. The existing literature examines the economic consequences of relative performance evaluation from two aspects: salary growth and salary stickiness.

Xiao and Peng [13] found that the ratcheting effect of rewards based on the total stock return performance of the same-scale enterprises in the same industry exists. The relative performance evaluation model based on the total stock return performance of the same-scale enterprises in the same industry can be used as a reference performance evaluation model to avoid ratcheting and to increase the incentive efficiency of the reward contract. Jiang [14] analysis believes that if the company adopts a relative performance evaluation, then the board of directors uses the average or median of similar company manager compensation to determine the company's managerial compensation. When the public companies in the market are widely adopted, they will continue to push up the average or median of similar company managers' compensation. The increase in the benchmark will prompt the board to continuously improve the manager's salary and will continue to push up the average or median of the pay of similar com- 
pany management. Through the test of the above theoretical analysis, it is found that the listed companies in China adopt the industry salary benchmark when formulating the manager compensation contract, which leads to the gradual increase of the manager's salary.

\section{Comments and Outlook}

Regarding the test of the existence of relative performance evaluation, foreign studies have drawn relatively consistent conclusions. However, due to the differences in research objects, reference companies' selection criteria and performance indicators, the domestic research results are still very different. How to comprehensively consider the influencing factors in practical application and construct the testable variables is the key to empirical research on relative performance evaluation. Specifically, it is a key issue to select which characteristics to define the reference enterprise and what type of performance indicators to choose as the basis for relative performance evaluation. Research on the influencing factors of relative performance evaluation has been rich, which can be classified into two groups: external influence factors and internal influence factors. External factors mainly include marketization process, market competition level, other subsidiaries within the group, etc. Internal factors mainly include ex ante promotion probability, management power, property rights, and growth. However, there is currently no analytical framework with intrinsic logic to integrate these factors to systematically understand the influencing factors of relative performance evaluation. Research on the economic consequences of relative performance evaluation is very scarce in China compared to foreign countries'. In summary, regarding the study of relative performance evaluation, foreign scholars are currently focusing on further research on the reasonable reference enterprise selection criteria and the economic consequences of relative performance evaluation, while domestic research is still based on traditional industry classification criteria to study the existence and influencing factors and research on the economic consequences of relative performance evaluation are still rare. Therefore, future research can focus on the following aspects.

\subsection{Consider Heterogeneity and Research Methods}

Further research should subdivide the type of enterprises to investigate the existence of the relative performance evalutation. Domestic research that finds no evidence of relative performance evaluation is mostly based on a full sample of all kinds of firms, but this does not indicate that there is no relative performance evaluation for different types of companies. Based on the existing research, future research should consider companies of sub-sectors (main board, small and medium-sized board, GEM), sub-industries (manufacturing, service industry, etc.) to investigate their probability of adopting relative performance evaluation. For example, companies of small and medium-sized board are mostly high-tech companies, and their risks are relatively higher. Compared with listed companies 
on the main board, small and medium-sized board companies are small in scale, and executives hold more shares and their shares are more concentrated. Whether this feature affects the application of relative performance evaluation remain to be further investigated. In addition, since the empirical research method may have deviations in simulating the application of the relative performance evaluation process by the board of directors, the research method should not be limited to the empirical research method. Various research methods such as questionnaire survey and experimental research method can be used to verify the existence and influencing factors of relative performance evaluation.

\subsection{Focus on Reasonable Peer Firms Selection Criteria}

Future research should also study reasonable selection criteria for peer firms. On the one hand, textual analysis can be widely applied in various public available materials of listed companies such as public disclosure of major issues, management analysis and discussion, brokerage research reports in order to refine certain important characteristics to fully reflect the common risks faced by specific enterprises. In addition to selecting a reference company based on the life cycle, it is also possible to refer to the benchmark analysis in the core competency assessment, taking into account internal benchmarks, competitive benchmarks, process or activity benchmarks, general benchmarks, customer benchmarks, and combining their own operating conditions and industry characteristics to determine reasonable peer firms selection criteria.

\subsection{Construct an Analytical Framework for Influencing Factors}

Establishing an analytical framework for influencing factors with intrinsic logical relationships is also necessary. Empirical studies show that external factors mainly include marketization process, market competition degree, other subsidiaries within the group, etc. Internal factors mainly include ex ante promotion probability, management power, ownership, corporate growth, and stock option grant. However, the current empirical research appears to be too fragmented and lacks an analytical framework with intrinsic logic. For example, what is the relationship between these influencing factors? Is it mutual promotion or suppression or even irrelevant? How do these factors affect the use of relative performance evaluation? Further categorizing these influencing factors according to specific criteria and analyzing the intrinsic relationship between each factor is the direction for further research.

\subsection{Further Examine the Economic Consequences of Relative Performance Evaluation}

On the one hand, the study of the economic consequences of relative performance evaluation must be based on a large number of empirical studies which proved the existence of the relative performance evaluation. On the other hand, few literatures study the economic consequences of relative performance evaluation from the perspective of earnings management and aggressive investment. 
However, because the relative performance evaluation eliminates the systematic part of the performance, the manager cannot make use of the external common favorable conditions to promote the company's performance growth. Therefore, in order to make the company's performance exceed the reference company's performance, managers may be inclined to carry out earnings management or aggressive investment, which is the direction of further research in the future.

\section{Conflicts of Interest}

The author declares no conflicts of interest regarding the publication of this paper.

\section{References}

[1] Jayaraman, S., Milbourn, T.T., Peters, F.S., et al. (2015) Product Market Peers and Relative Performance Evaluation. Social Science Electronic Publishing.

[2] Drake, K.D. and Martin, M. (2016) Implementing Relative Performance Evaluation: The Role of Life Cycle Peers. Social Science Electronic Publishing.

[3] Dikolli, S.S., Diser, V., Hofmann, C., et al. (2018) CEO Power andmance evaluation. Contemporary Accounting Research, 35, 1279-1296. https://doi.org/10.1111/1911-3846.12316

[4] Hu, Y. and Zhou, H. (2012) Executive Compensation, Corporate Growth Level and Relative Performance Evaluation-Evidence from Chinese Listed Companies. Accounting Research, No. 5, 22-28.

[5] Chen, D., Liang, S. and Zhu, P. (2012) Relative Performance Evaluation and Executive Compensation: Evidence from Chinese Listed Companies. China Journal of Accounting Research, 5, 127-144. https://doi.org/10.1016/j.cjar.2012.02.002

[6] Zhou, H., Jian, L., Li, G., et al. (2014) Competition Degree and Relative Performance Evaluation in Different Markets-Based on the Empirical Test of Shanghai and Shenzhen Listed Companies from 2004 to 2012. Accounting Research, No. 5, 59-66.

[7] Zhang, D. and Wang, Y. (2016) Marketization Process, Executive Compensation and Relative Performance Evaluation-Evidence from Shanghai and Shenzhen A Shares. Journal of Jinan University (Philosophy and Social Sciences), 38, 117-125.

[8] Cai, G. and Zheng, G. (2016) Executive Compensation in Business Groups: Evidence from China. China Journal of Accounting Research, 9, 25-39. https://doi.org/10.1016/j.cjar.2015.06.003

[9] Mahlendorf, M.D., Kleinschmit, F. and Perego, P. (2014) Relational Effects of Relative Performance Information: The Role of Professional Identity. Accounting, Organizations and Society, 39, 331-347. https://doi.org/10.1016/j.aos.2014.05.001

[10] Park, H. and Vrettos, D. (2015) The Moderating Effect of Relative Performance Evaluation on the Risk Incentive Properties of Executives' Equity Portfolios. Journal of Accounting Research, 53, 1055-1108. https://doi.org/10.1111/1475-679X.12092

[11] Angelis, D. and Grinstein, Y. (2016) Relative Performance Evaluation in CEO Compensation: A Non-Agency Explanation. Social Science Electronic Publishing.

[12] Demeré, B.W., Krishnan, R., Sedatole, K.L., et al. (2016) Do the Incentive Effects of Relative Performance Measurement Vary with the Ex Ante Probability of Promotion? Management Accounting Research, 30, 18-31.

https://doi.org/10.1016/j.mar.2015.09.002 
H. H. Li

[13] Xiao, J. and Peng, W. (2010) The Ratcheting Effect of Managerial Compensation and the Choice of Relative Performance Evaluation Model. Chinese Journal of Management, 7, 993-999.

[14] Jiang, W. (2010) Industry Salary Benchmark and Managerial Salary Growth-An Empirical Analysis Based on Chinese Listed Companies. Financial Research, No. 4, 144-159. 